\title{
Kinetic study on the sorption and degradation of antibiotics in the estuarine water: an evaluation based on single and multiple reactions
}

\author{
Jia $\mathrm{Li}^{1,2} \cdot \operatorname{Min} \mathrm{Cui}^{1}$ \\ Received: 23 April 2020 / Accepted: 20 July 2020 / Published online: 24 July 2020 \\ (C) Springer-Verlag GmbH Germany, part of Springer Nature 2020
}

\begin{abstract}
It is important to study the fate and transport of antibiotics in aquatic environments to reveal their pollution status. The premise behind fate and transport studies is to evaluate the reaction processes of the target antibiotics. However, available research on the environmental behaviors of antibiotics in certain natural waters, such as estuarine water, is scarce. In this study, single reactions such as sorption, biodegradation, and photolysis and multiple degradation reactions of sulfamethoxazole (SMX), trimethoprim (TMP), and ciprofloxacin (CIP) in the estuarine water were studied. The sorption rates of the target antibiotics in the estuarine water-sediment system were very fast, and the sorption amounts varied among sediments and antibiotics. Hydrolysis did not contribute to the degradation of the target antibiotics. Biodegradation had a low contribution to the degradation of the target antibiotics in the estuarine water. In comparison, photolysis was the dominant degradation process for SMX, TMP, and CIP. The rates of photolysis of the tested antibiotics in the estuarine water were greater than those in pure water; thereby, indicating photolysis of these antibiotics was more prone to occur in the estuarine water. In the multiple degradation experiments, it was found that there may be synergistic effects between the single degradation processes. Thus, the aqueous concentrations of antibiotics decreased rapidly by sorption after entering the estuarine water and then decreased relatively slowly by photolysis and biodegradation. This study provides information for evaluating the environmental behaviors of antibiotics in estuarine environments.
\end{abstract}

Keywords Antibiotics $\cdot$ Kinetics $\cdot$ Sorption $\cdot$ Hydrolysis $\cdot$ Photolysis $\cdot$ Biodegradation $\cdot$ Estuarine water

\section{Introduction}

Antibiotics are becoming increasingly essential in modern society owing to their superior efficiency in treating diseases and

Responsible Editor: Ester Heath

Electronic supplementary material The online version of this article (https://doi.org/10.1007/s11356-020-10194-4) contains supplementary material, which is available to authorized users.

Jia Li

lijia3611@yzu.edu.cn

1 School of Environmental Science and Engineering, Yangzhou University, Yangzhou 225127, China

2 Key Laboratory of Coastal Environmental Process and Ecology Remediation, Yantai Institute of Coastal Zone Research, Chinese Academy of Sciences; Shandong Key Laboratory of Coastal Environmental Processes, YICCAS, Yantai 264003, China promoting animal growth. With the global increase in demand for animal protein for human consumption, the global consumption of veterinary antibiotics is expected to increase by 67\% from 2010 to 2030 (Van Boeckel et al. 2015). The extensive consumption of antibiotics increases the risk that large amounts of residual antibiotics will end up in the environment because most antibiotics are poorly absorbed by humans and animals (Zhu et al. 2013). Antibiotic concentrations ranging from $\mathrm{ng} / \mathrm{L}$ to $\mathrm{mg} / \mathrm{L}$ have been reported in various environmental media, such as wastewater, rivers, lakes, seawater, sludge, sediment, and soil (Li et al. 2012; Zhang et al. 2013; Guerra et al. 2014; Cheng et al. 2019; Ngigi et al. 2020). These residual antibiotics in environments are important in selection of antibiotic-resistant bacteria (ARBs) and antibiotic resistance genes (ARGs) (Huijbers et al. 2020; Zhu et al. 2013). Luo et al. (2010) reported that the relative abundance of the ARGs (i.e., sul1/16S-rDNA and sul2/16S-rDNA) in the Haihe River is positively related with the total concentration of sulfonamides. Further, antibiotics can cause adverse effects 
on microbes, animals, and plants and accumulate along food webs (Bialk-Bielinska et al. 2011; Liu et al. 2017). The pollution caused by antibiotics and their ecological toxic effects has attracted international attention.

After entering the aquatic environment, antibiotics may undergo long-range transport. As reported by Biel-Maeso et al. (2018), antibiotics in the effluents of wastewater treatment plants (WWTPs) can be transferred from the river to the estuary and then to the bay and eventually to the open sea. Nevertheless, antibiotics are not persistent pollutants. Under the combined effects of physical, chemical, and biological actions, antibiotics may undergo a series of reactions such as photolysis, hydrolysis, biodegradation, mineralization, and sorption/desorption (Homem and Santos 2011; Charuaud et al. 2019). It is well established that these biogeochemical reactions combined with water dilution will cause attenuation of antibiotics. In order to reveal the fate and transport of antibiotics in surface water, reactive transport models (e.g., WASP, PhATE, GREAT-ER) have been developed, optimized, and used (Schowanek and Webb 2002; Hosseini et al. 2012; Arlos et al. 2014). The accuracy of the simulation results depends on the model parameters, especially the parameters of the key reactions. Arlos et al. (2014) reported that the reaction rate constants (e.g., photolysis rate constant) of pharmaceuticals can improve the model performance. Therefore, studying the reactive processes of antibiotics and calculating their degradation rate constants will provide reference data for modeling.

The reaction constants of the same antibiotic in different aqueous matrixes may have large differences. Biosic et al. (2017) reported that the photodegradation rate constant of sulfamethazine in synthetic wastewater is four times greater than that in pure water. Our previous study also showed that the distribution coefficient of the same antibiotic in pure water was different from that in seawater ( $\mathrm{Li}$ et al. 2018). Furthermore, the reaction process varies among various types of antibiotics (Alexy et al. 2004; Adamek et al. 2016). That is, the specific reaction (e.g., sorption, hydrolysis, photolysis, and biodegradation) of the target antibiotics in the tested matrix should be investigated to provide accurate reaction rate constants for modeling. Among the above reactions, sorption/desorption of antibiotics has received more attention. However, most studies focused on the sorption/desorption of antibiotics in artificial water systems. Similarly, the degradation of antibiotics in natural waters has been less studied, and the corresponding reaction coefficients are relatively difficult to obtain for certain antibiotics. The few studies addressing this subject are limited to freshwater (Adamek et al. 2016; Caracciolo et al. 2018; Zhuang et al. 2019).

Estuaries are an important route for the transport of terrestrial material to the ocean (Simpson et al. 2001). Under the combined effects of river runoff and tides, salinity, $\mathrm{pH}$, redox potential, and the concentration of particles exhibit high spatial and temporal variability (Chapman and Wang 2001). The unique hydrological properties may affect the sorption and degradation of antibiotics in estuarine environments and influence the spatial and temporal distribution characteristics of antibiotics as well as their ecological risks. However, the fate and transport behaviors of antibiotics in estuarine environments remain poorly understood. There is an urgent need to develop and conduct simulation studies of antibiotics in estuarine water.

The Xiaoqing River (XQR) is a major pollution source in Laizhou Bay. Intensive anthropogenic activities have released a large amount of pollutants into the XQR. Owing to the special hydrogeomorphic features, pollutants are prone to be stranded in the XQR estuary for a long period of time. Our previous investigation showed that the XQR water was polluted by multiple kinds of antibiotics such as sulfonamides, sulfonamide synergists, and fluoroquinolones (Li et al. 2020). Based on the investigation results, three antibiotics (i.e., sulfamethoxazole (SMX), trimethoprim (TMP), and ciprofloxacin (CIP)) with maximum concentrations exceeding $800 \mathrm{ng} / \mathrm{L}$ and/or with high ecological risks (RQs $>1.0$ ) were selected as the targets. These three antibiotics have been detected in estuarine environments worldwide. Thus, this study provides information for fate modeling of the three antibiotics in estuarine systems.

The objective of this study was to reveal the sorption kinetics and degradation processes of SMX, TMP, and CIP in natural estuarine water. To this end, indoor simulation experiments were conducted to (1) study the sorption, photolysis, and biodegradation of three antibiotics in the estuarine water; (2) study the hydrolysis of the target antibiotics under different $\mathrm{pH}$ conditions; and (3) evaluate the degradation rate constants of antibiotics based on single and multiple reactions.

\section{Materials and methods}

\section{Chemicals}

The antibiotic standards including SMX, TMP, and CIP were purchased from Sigma-Aldrich Company (USA). The physicochemical properties of the three antibiotics are shown in Table S1. Methanol and acetonitrile were HPLC grade and were obtained from Anaqua Chemicals Supply (ACS, USA). The other chemicals and solvents used in this study were of analytical grade or above. Antibiotic was dissolved in methanol to prepare the stock solution (S1). These stock solutions were stored at $4{ }^{\circ} \mathrm{C}$ until used. Working solutions of each antibiotic with a concentration of $1.0 \mathrm{mg} / \mathrm{L}$ were prepared by diluting the stock solution using a background solution. 


\section{Background solution and sediments}

Background solution used in this study includes buffer solution, the estuarine water, and ultrapure water. Among them, buffer solution was used for single hydrolysis experiments; the estuarine water was used for sorption kinetics experiments, single photolysis, single biodegradation experiments, and multiple degradation experiments; further, ultrapure water was also prepared for photolysis experiments. Buffer solutions with the $\mathrm{pH}$ value of $4.0,7.0$, and 9.0 were prepared using the method by Biosic et al. (2017). The estuarine water was collected from the XQR estuary $\left(37^{\circ} 11^{\prime} 08.13^{\prime \prime}\right.$ north latitude and $118^{\circ} 34^{\prime} 10.92^{\prime \prime}$ east longitude). Physicochemical properties of the estuarine water are analyzed and shown in Table S2. Before conducting experiments, the filtered estuarine water was measured as a blank, and the background concentrations of the target antibiotics were lower than their detection limits. Ultrapure water (MQ) was obtained from a Milli-Q water purification system (Millipore, Billerica, MA, USA). Two sediments represent to high content of organic carbon (sediment $\mathrm{H}$ ) and low content of organic carbon (sediment $\mathrm{L}$ ) were selected from the XQR estuary. The sediment samples were freeze-dried. After removing the gravels, sediment samples were ground into particles and then pass through a 60-mesh sieve. The detail information of sediment samples is described in Table S3.

\section{Sorption kinetics experiments}

A $0.2 \mathrm{~g}$ of sediment sample was placed into a $250 \mathrm{~mL}$ of conical flask. Then, $50 \mathrm{~mL}$ of antibiotic working solution $(1.0 \mathrm{mg} / \mathrm{L})$ containing $50 \mathrm{mg}$ of mercuric chloride was added. Parafilm was used to seal the flask. The flasks were shaken at $25^{\circ} \mathrm{C}$ in a temperature-controlled shaking incubator (Harbin, China) at a shaking speed of $190 \mathrm{rpm}$. The shaking incubator was covered with black cloth aim to prevent the occurrence of photodegradation. A $1.0 \mathrm{~mL}$ sample was taken at $0,1,3,6,12$, 24,36 , and $48 \mathrm{~h}$. The sample was filtered through a $0.22-\mu \mathrm{m}$ syringe filter before analysis. Two blank sorption experiments, one with the reactor system containing antibiotic without sediment and another containing sediment without antibiotic, were carried out. All the sorption experiments were conducted in triplicate. Each antibiotic was tested separately.

\section{Single hydrolysis experiments}

To initiate the single hydrolysis experiments, $50 \mathrm{~mL}$ of antibiotic working solution $(1.0 \mathrm{mg} / \mathrm{L})$ prepared using buffer solution ( $\mathrm{pH} 4.0,7.0,9.0$ ) was added to a $50-\mathrm{mL}$ glass vial, followed by adding $50 \mathrm{mg}$ of mercuric chloride. The glass vials were wrapped using aluminum foil and stored at $25^{\circ} \mathrm{C}$. A $1.0 \mathrm{~mL}$ sample was taken at $0,1,3,10,14,21,28,42$, and 56 days and filtered through a $0.22-\mu \mathrm{m}$ syringe filter before analysis. All the hydrolysis experiments were conducted in triplicate. Each antibiotic was tested separately.

\section{Single photolysis experiments}

Single photolysis experiments were conducted at $25^{\circ} \mathrm{C}$, by adding $50 \mathrm{~mL}$ of antibiotic working solution of $1.0 \mathrm{mg} / \mathrm{L}$ into a $50-\mathrm{mL}$ glass vial with $50 \mathrm{mg}$ of mercuric chloride. The working solution was prepared in the estuarine water and ultrapure water, respectively. Tighten the cap, and then, put the glass vials into an illumination incubator (Shedrock, China) equipped with a high-pressure mercury lamp. The light intensity is set to 3500 Lux. Light cycle is $12 \mathrm{~h}$ of light and $12 \mathrm{~h}$ of dark. The vessels of control experiment were wrapped by aluminum foil and then incubated under the same conditions to those described above. A $1.0 \mathrm{~mL}$ sample was taken at 0,1 , $3,7,14,21,28,42$, and 56 days. Samples were filtered through a $0.22-\mu \mathrm{m}$ syringe filter before analysis. All the photolysis experiments were conducted in triplicate. Each antibiotic was tested separately.

\section{Single biodegradation experiments}

Single biodegradation experiments were also carried out at $25^{\circ} \mathrm{C}$ in an illumination incubator. First, $50 \mathrm{~mL}$ of antibiotic working solution $(1.0 \mathrm{mg} / \mathrm{L})$ prepared in the estuarine water was added to a $50-\mathrm{mL}$ glass vial. The same amount of antibiotic working solution and $50 \mathrm{mg}$ of mercuric chloride were added in the control experiments. All the vessels were wrapped using aluminum foil. A $1.0 \mathrm{~mL}$ sample was taken at $0,1,3,10,14,21,28,42$, and 56 days. All the biodegradation experiments were conducted in triplicate. Each antibiotic was tested separately. Before the experiment, the number of microbes in the estuarine water of the experimental and control groups was measured using the plate count method. Briefly, a $100 \mu \mathrm{L}$ sample of the estuarine water was coated on a plate containing tryptose soya agar ( $90 \mathrm{~cm}$ in diameter) and then incubated for $48 \mathrm{~h}$ at $35^{\circ} \mathrm{C}$. The total number of bacterial colonies on the plate was counted by eye.

\section{Multiple degradation experiments}

Antibiotic working solution prepared in the estuarine water was used to conduct the multiple degradation experiments. The experimental procedures are same with the single photolysis experiments, except that the mercuric chloride was not added.

\section{Measurements and data analyses}

The target antibiotics were detected using ultra-performance liquid chromatography (UPLC) equipped with a photo-diode array detector (PDA), an ACQUITY UPLC BEH C18 column 
$(2.1 \times 50 \mathrm{~mm}, 1.7 \mu \mathrm{m})$, and a $2.1 \times 5 \mathrm{~mm}$ VanGuard pre-column. The column temperature was $30^{\circ} \mathrm{C}$. Injection volume was $10 \mu \mathrm{L}$. Mobile phase A was ultrapure water with $1.0 \%$ acetic acid and $\mathrm{B}$ was acetonitrile. The proportion of mobile phase, detection wavelength, and detection limit of the three antibiotics is shown in Table S4. The relative standard deviation (RSD) of the test results was less than $0.3 \%$. For each antibiotic, two standard curves (one for ultrapure water and another for estuarine water) with six-point (i.e. 0.1, 0.25, 0.5, $0.75,1$, and $1.5 \mathrm{mg} / \mathrm{L})$ were used for quantitative analysis. The $R^{2}$ values of standard curves are higher than 0.990 .

Degradation kinetics of antibiotics is fitted with the firstorder kinetic reaction equation (Eq. (1)).

$C_{t}=C_{0} \times e^{-k t}$

where $C_{0}$ and $C_{t}$ are the concentration of antibiotics at time 0 and time $t(\mathrm{~d})$, and $k$ is the degradation rate constant (/d). When $C_{t}=0.5 C_{0}$, the half-life $\left(t_{0.5}\right)$ of antibiotics is shown in Eq. (2).

$t_{0.5}=\ln 2 / k$

Pseudo-first-order kinetic model (Eq. (3)) and pseudosecond-order kinetic model (Eq. (4)) are used to obtain information on sorption kinetics of antibiotics:

$q_{t}=q_{e}\left(1-e^{-k_{1} t}\right)$

$\frac{t}{q_{t}}=\frac{t}{q_{e}}+\frac{1}{k_{2} q_{e}^{2}}$

where $q_{t}(\mathrm{mg} / \mathrm{g})$ and $q_{e}(\mathrm{mg} / \mathrm{g})$ are the sorption amounts of antibiotic at time $t(\mathrm{~h})$ and at equilibrium; $k_{1}(/ \mathrm{h})$ and $k_{2}$ $((\mathrm{g} / \mathrm{mg}) / \mathrm{h})$ are rate constants for pseudo-first order and pseudo-second order, respectively.

Data analysis was performed using Microsoft Excel 2016 and OriginPro 2015 (OriginLab Corporation, Northampton, MA). Statistical analysis was performed using the SPSS software (Version 19.0, IBM-SPSS, Chicago, IL, USA). An analysis of variance (ANOVA) and Student's $t$ test were performed for evaluating the differences of data obtained from various experimental groups, and the significance level was set at $p<0.05$.

\section{Results and discussion}

\section{Sorption kinetics of antibiotics}

The sorption kinetics of the antibiotics on the two sediments are shown in Fig. 1. The sorption of the three antibiotics was fast in the initial $1 \mathrm{~h}$, especially for CIP, which accounted for $93.7 \%$ of the total sorption amount for sediment L and $95.8 \%$ of that for sediment $\mathrm{H}$. This was attributed to the high concentration gradient at the beginning of the sorption process, which exhibited a strong driving force for the transport of antibiotics from the solution to the surface of sediments particles. The sorption rate then decreased slowly until reaching the sorption equilibrium. The sorption equilibriums of SMX and TMP on sediment were generally achieved within $36 \mathrm{~h}$, while the sorption equilibrium of CIP was achieved in only $12 \mathrm{~h}$. This indicated that sorption of CIP was easier and faster than that of the other two antibiotics on the same sediment. This might have been related to their characteristics. As shown in Table S1, the $\log K_{\text {ow }}$ of CIP (1.32) is higher than that of SMX (0.89) and TMP (0.91), indicating that CIP was more likely to be adsorbed. Moreover, all the tested antibiotics were zwitterions and anions in the reaction systems $(\mathrm{pH}=7.4)$, but CIP also contained a portion of cations. The sediment particles carried the negative charge because the $\mathrm{pH}$ of reaction systems was higher than the $\mathrm{pH}_{\mathrm{pzc}}$ (i.e., $\mathrm{pH}$ at point of zero charge) values of the sediments (Table S3). In this case, the cations of CIP enhanced its sorption capacity on the negatively charged sediment particles through electrostatic attraction. The sorption amounts of all the antibiotics on sediment $\mathrm{H}$ were slightly (ANOVA; $p>0.05$ ) greater than those on sediment L. This was because sediment with a high organic matter and/or ironaluminum oxides has a stronger sorption capacity for antibiotics (Cao et al. 2015; Li and Zhang 2016; Riaz et al. 2018).

The experimental data were fitted into kinetic models to quantify the sorption rates of the antibiotics on the sediments. The kinetic model parameters are summarized in Table 1. It was observed that CIP sorption onto sediments followed both the pseudo-first-order and pseudo-second-order reactions with indistinguishable correlation coefficients. For SMX and TMP, the $R^{2}$ values were relatively higher for the pseudo-secondorder model than for the pseudo-first-order model. Additionally, the sorption capacity $\left(q_{e, \mathrm{cal}}\right)$ obtained from the pseudo-second-order kinetic model was much closer to the experimental data $\left(q_{e, \text { exp }}\right)$. Conformance to the pseudosecond-order model suggested that the process controlling the rate may be chemical sorption (Wang et al. 2010).

As shown in Table 1, the sorption rate constants of the three antibiotics on sediment $\mathrm{L}$ are higher than those on sediment $\mathrm{H}$. Compared with sediment $\mathrm{L}$, sediment $\mathrm{H}$ had a higher content of iron-aluminum oxides (Table S3). The organic matter content in sediment $\mathrm{H}(1.69 \%)$ was also higher than that in sediment $\mathrm{L}$ $(0.385 \%)$. That is, there were more available sorption sites on sediment H, which could adsorb more antibiotics. Therefore, more time was needed to achieve sorption equilibrium for the sorption of antibiotics on sediment $\mathrm{H}$. This result was consistent with a previous study that reported that the sorption rates of antibiotics on soils with higher contents of organic matter and iron-aluminum oxides are relatively slow (Fernández-Calviño et al. 2015). Further, our previous study showed that the sorption rate constant of TMP on sediments is negatively related to the clay content ( $\mathrm{Li}$ and Zhang 2017). These results indicate that sediment properties (e.g., organic matter, particle size) 


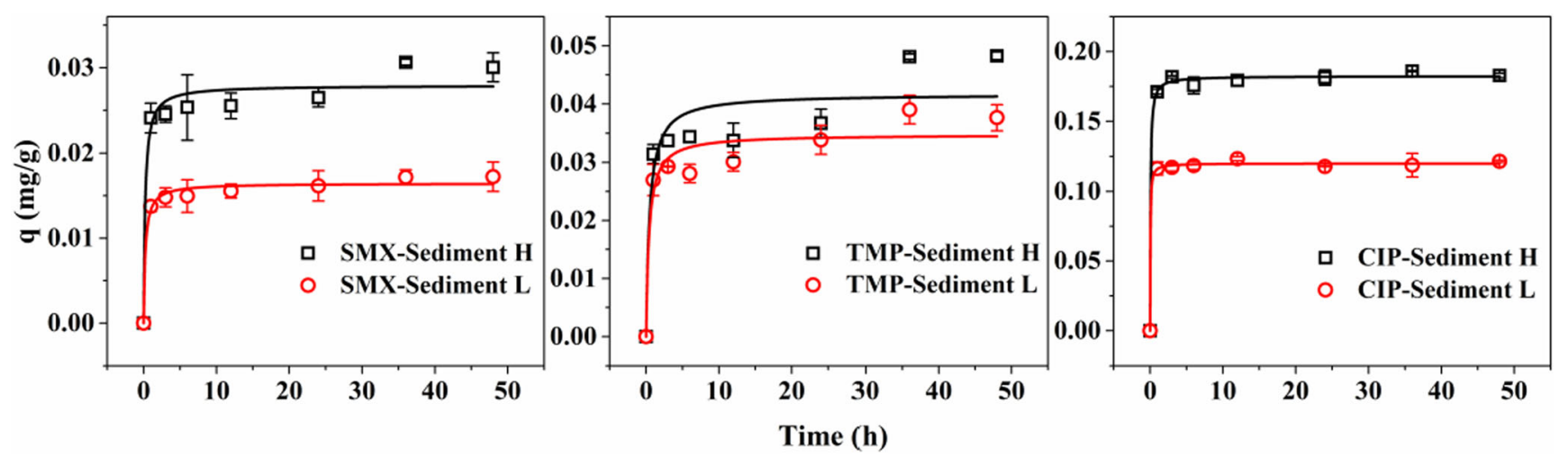

Fig. 1 Sorption kinetics of antibiotics onto sediments. Solid lines are pseudo-second-order kinetic model simulations

affect sorption kinetics of the target antibiotics. Thus, it would be beneficial to consider the sediment properties when choosing the sorption rate constants of antibiotics.

\section{Hydrolysis of antibiotics}

The hydrolysis processes of the three antibiotics were studied under different $\mathrm{pH}$ ( $\mathrm{pH}$ of 4.0, 7.0, 9.0) conditions. As shown in Fig. 2, 56 days of hydrolysis had little or no effect on the concentrations of the three antibiotics. SMX was stable at a $\mathrm{pH}$ of 7.0 and 9.0, while a weak hydrolysis reaction occurred at a $\mathrm{pH}$ of 4.0 , where less than 5\% of SMX was degraded. Our results were consistent with a previous study (Bialk-Bielinska et al. 2012), which reported that the hydrolysis of SMX at a $\mathrm{pH}$ of 4.0 was higher than that at a $\mathrm{pH}$ of 7.0 and 9.0. They attributed this to the fact that the presence of cationic forms of sulfonamides in acidic solution is more sensitive to hydrolysis than the neutral and anionic forms of these compounds. TMP and CIP hardly undergo hydrolysis reaction under the three $\mathrm{pH}$ conditions. As summarized by Norvill et al. (12016), fluoroquinolone and sulfonamide antibiotics do not break down via hydrolysis under normal environmental conditions. This is because these antibiotics do not possess structural features that can be readily hydrolyzed under environmental conditions (Loftin et al. 2008). In other words, the hydrolysis of fluoroquinolones and sulfonamides requires higher temperatures, and higher concentrations of strong acids or bases than may be encountered in the environment. Therefore, the hydrolysis of SMX, CIP, and TMP is negligible in natural water.

\section{Photolysis of antibiotics}

As is well known, the photolysis of chemical substances includes direct photolysis, sensitized photolysis, and indirect photolysis. Direct photolysis can occur by absorbing light energy, whereas sensitized photolysis and indirect photolysis require the help of other natural substances in the solvent. That is, photolysis depends on the matrix composition. In order to evaluate the impact of the matrix composition on the photolysis processes of the target antibiotics, photolysis experiments were conducted in the estuarine water and ultrapure water. The concentrations of the three antibiotics in both aqueous matrixes decrease apparently after 56 days of photolysis (Fig. 3), indicating noticeable photodegradation. The photolysis of antibiotics in the estuarine water was greater than that in ultrapure water. One-way ANOVA indicates that the photolysis rate

Table 1 Kinetic model parameters for sorption of antibiotics on river sediments

\begin{tabular}{|c|c|c|c|c|c|c|c|}
\hline \multirow[t]{2}{*}{ Sediment } & \multirow[t]{2}{*}{$q_{(e, \exp )}(\mathrm{mg} / \mathrm{g})$} & \multicolumn{3}{|c|}{ Pseudo-first-order kinetic model } & \multicolumn{3}{|c|}{ Pseudo-second-order kinetic model } \\
\hline & & $q_{(e, \text { cal })}(\mathrm{mg} / \mathrm{g})$ & $k_{1}(/ \mathrm{h})$ & $R^{2}$ & $q_{(e, \mathrm{cal})}(\mathrm{mg} / \mathrm{g})$ & $k_{2}((\mathrm{~g} / \mathrm{mg}) / \mathrm{h})$ & $R^{2}$ \\
\hline \multicolumn{8}{|l|}{ CIP } \\
\hline Sediment $\mathrm{H}$ & 0.179 & 0.181 & $2.90 \pm 0.341$ & 0.998 & 0.182 & $85.3 \pm 28.8$ & 0.998 \\
\hline Sediment L & 0.123 & 0.120 & $3.65 \pm 0.751$ & 0.997 & 0.120 & $235 \pm 138$ & 0.998 \\
\hline \multicolumn{8}{|l|}{ TMP } \\
\hline Sediment $\mathrm{H}$ & 0.048 & 0.039 & $1.51 \pm 0.781$ & 0.817 & 0.042 & $50.2 \pm 34.9$ & 0.860 \\
\hline Sediment L & 0.039 & 0.033 & $1.62 \pm 0.670$ & 0.884 & 0.035 & $70.8 \pm 38.9$ & 0.920 \\
\hline \multicolumn{8}{|l|}{ SMX } \\
\hline Sediment H & 0.031 & 0.027 & $2.16 \pm 0.813$ & 0.941 & 0.028 & $166 \pm 95.9$ & 0.957 \\
\hline Sediment L & 0.017 & 0.016 & $1.93 \pm 0.442$ & 0.971 & 0.017 & $258 \pm 82.6$ & 0.985 \\
\hline
\end{tabular}



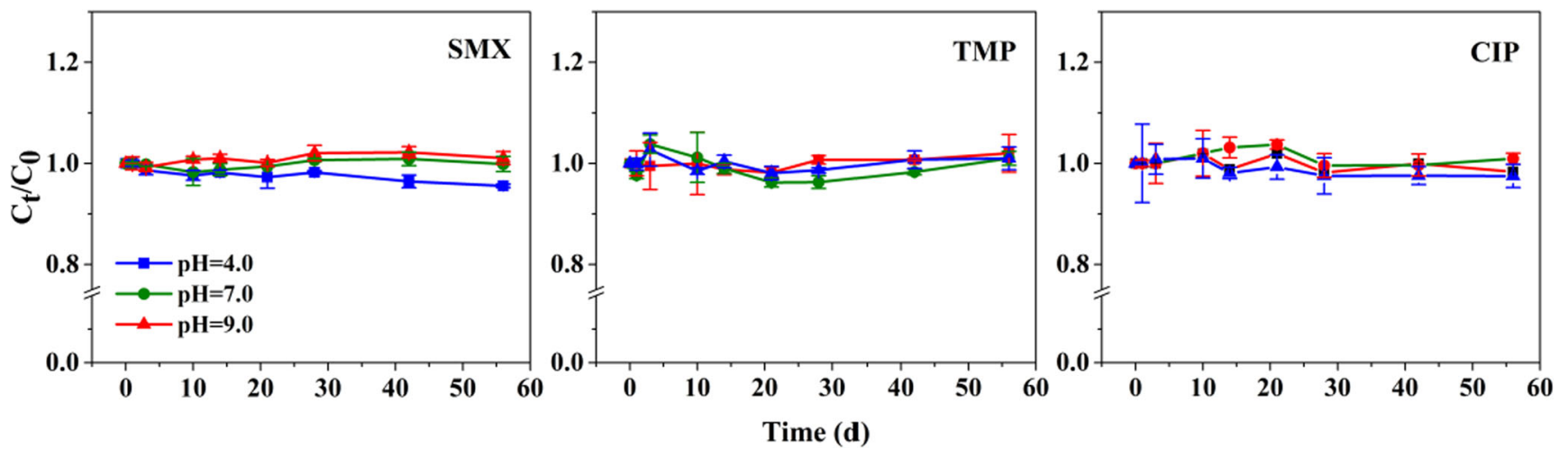

Fig. 2 Hydrolysis of antibiotics under different $\mathrm{pH}$ conditions

constants of the three antibiotics in the estuarine water are slightly higher $(p>0.05)$ than that in the ultrapure water (Table 2). These results were consistent with the results of a previous study which reported that the rate of sulfamethazine photolysis in different media was in the order of wastewater $>$ river water $>$ pure water (Garcia-Galan et al. 2012). The greater photolysis of SMX, TMP, and CIP in the estuarine water might have been due to the enhancement of indirect photolysis. There are many important promoters of antibiotic photolysis, such as NO- 3 and dissolved organic matter, in natural waters (Lin and Reinhard 2005; Tong et al. 2011; Xu et al. 2011). Another possible reason may be that the photolysis of antibiotics is related to the ion strength. As reported by Zhang et al. (2016), more effective collisions between reactants occurred under the high ion strength condition, which in turn accelerated the photodegradation rate. The conductivity of the estuarine water $(3070 \mu \mathrm{S} / \mathrm{cm})$ was higher than that of the ultrapure water $(2.30 \mu \mathrm{S} / \mathrm{cm})$; thereby, a fast photolysis of antibiotics occurred in the estuarine water. Further, Biosic et al. (2017) reported that chloride ions have various impacts on the photodegradation of different types of antibiotics. It is well known that the concentration of chloride ions in estuarine waters is pretty high. Hence, the effect of chloride ions on the photolysis behavior of various antibiotics must to be investigated on a case-by-case basis in future studies.
As shown in Table 2, the photolysis rates in the same aqueous matrixes vary among the antibiotics. In both the estuarine water and ultrapure water, the photolysis rates of the three antibiotics were in the order of CIP > SMX $>$ TMP. Alharbi et al. (2017) found that SMX is more prone to photolysis than TMP. Choi et al. (2016) also reported that the photolysis extent of SMX is greater than that of TMP, while the photolysis of both antibiotics is much weaker than that of fluoroquinolones. This is possibly due to the extremely symmetric structure (i.e., stable structure) of TMP, which results in degradation persistence (Choi et al. 2016). The piperazine ring of CIP is prone to cleavage, thereby leading to the enhancement of its photodegradation (Cardoza et al. 2005). In addition, the photolysis rates of the three antibiotics obtained from our study were different from those reported in the literature. For example, Caracciolo et al. (2018) showed that the photolysis half-life of CIP in the river water is 18.4 days, which is faster than that found in our study (27 days). According to Gmurek et al. (2015), the photolysis half-life of SMX in pure water is 7 days, which is far smaller than the result of our study (103.3 days). This may be caused by different experimental conditions (e.g., matrix composition, experimental light intensity).
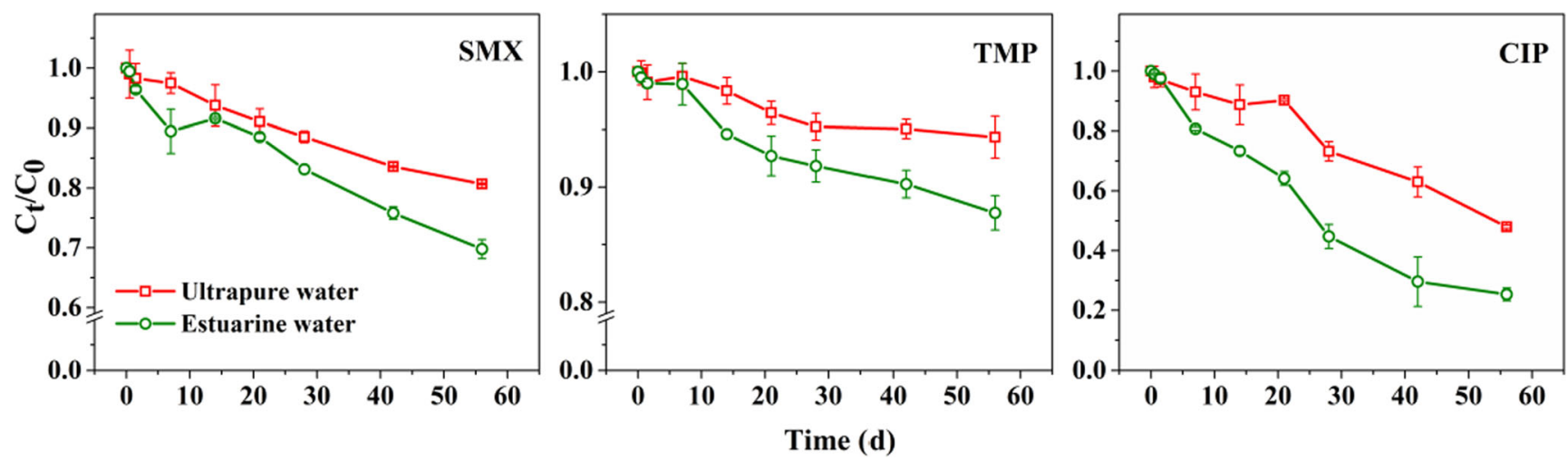

Fig. 3 Photolysis of antibiotics in the estuarine water and ultrapure water 
Table 2 Degradation rate constants of antibiotics under different experimental conditions

\begin{tabular}{lllll}
\hline Antibiotics & Matrix & $k(/ \mathrm{d})$ & $t_{0.5}(\mathrm{~d})$ & $R^{2}$ \\
\hline Photolysis & & & & \\
SMX & Ultrapure water & $4.11 \times 10^{-3} \pm 1.20 \times 10^{-4}$ & 168.6 & 0.987 \\
& Estuarine water & $6.71 \times 10^{-3} \pm 5.51 \times 10^{-4}$ & 103.3 & 0.912 \\
TMP & Ultrapure water & $1.23 \times 10^{-3} \pm 1.01 \times 10^{-4}$ & 563.5 & 0.889 \\
& Estuarine water & $2.61 \times 10^{-3} \pm 1.70 \times 10^{-4}$ & 265.6 & 0.930 \\
CIP & Ultrapure water & $1.09 \times 10^{-2} \pm 9.07 \times 10^{-4}$ & 63.7 & 0.936 \\
& Estuarine water & $2.57 \times 10^{-2} \pm 1.18 \times 10^{-3}$ & 27.0 & 0.986 \\
Biodegradation & & & \\
SMX & Estuarine water & $1.06 \times 10^{-3} \pm 1.05 \times 10^{-4}$ & 653.9 & 0.923 \\
TMP & Estuarine water & $1.10 \times 10^{-3} \pm 7.52 \times 10^{-5}$ & 630.1 & 0.892 \\
CIP & Estuarine water & $2.01 \times 10^{-3} \pm 2.93 \times 10^{-4}$ & 344.8 & 0.592 \\
Multiple reactions & & & \\
SMX & Estuarine water & $9.15 \times 10^{-3} \pm 5.29 \times 10^{-4}$ & 80.2 & 0.958 \\
TMP & Estuarine water & $4.89 \times 10^{-3} \pm 1.70 \times 10^{-4}$ & 141.7 & 0.962 \\
CIP & Estuarine water & $2.84 \times 10^{-2} \pm 1.18 \times 10^{-3}$ & 24.4 & 0.982 \\
\hline
\end{tabular}

\section{Biodegradation of antibiotics}

The biodegradation of the three antibiotics under aerobic conditions was studied in the estuarine water at $25^{\circ} \mathrm{C}$. The microbial count in the estuarine water was $5000 \pm 500 \mathrm{CFU} / \mathrm{mL}$, while no bacteria were found in the control group with added mercuric chloride. The removal efficiency of antibiotics through biodegradation was calculated according to the difference between the experimental group and the control group. As shown in Fig. 4, SMX and TMP are stable until day 27 , as demonstrated by the reductions in their initial concentrations of less than $2.5 \%$. A decrease of approximately $4 \%$ was observed for the two antibiotics. The decrease in the CIP concentration in the first 5 days was also not significant. The lag of biodegradation of antibiotics was also reported in earlier studies (Ingerslev and Halling-Sorensen 2000; Biosic et al. 2017). Biosic et al. (2017) stated that the microbiota likely did not adopt their enzymes to use antibiotics as nutrients at the beginning of the experiments. Therefore, the biodegradation of antibiotics is characterized by hysteresis. The CIP concentration increased from day 28 to day 42 . This could be attributed to the conversion between the parent compound and its metabolites. The conversion of the acetylated degradation products back to their parent form in various environmental matrixes (e.g., wastewater, manure) has been reported in the literature (Gobel et al. 2004).

According to the Student's $t$ test, the biodegradation rate constant of CIP $\left(2.01 \times 10^{-3} \pm 2.93 \times 10^{-4} / \mathrm{d}\right)$ is significantly higher $(p<0.05)$ than that of SMX $\left(1.06 \times 10^{-3} \pm 1.05 \times\right.$ $\left.10^{-4} / \mathrm{d}\right)$ and TMP $\left(1.10 \times 10^{-3} \pm 7.52 \times 10^{-5} / \mathrm{d}\right)$ (Table 2$)$, indicating that CIP was more prone to biodegradation. This might have been due to the unstable piperazine ring of CIP. Previous studies have reported that demethylation of the piperazine ring of CIP is its dominant biodegradation pathway (Amorim et al. 2014; Jia et al. 2018). The presence of $-\mathrm{NH}_{2}$ and $-\mathrm{CH}_{3}$ in the molecular structure of SMX and TMP should improve their biodegradability. However, the opposite result was obtained in our study. This was probably due to the microbial community structure of the estuarine water. The biodegradation of an antibiotic depends on the presence of microbial populations that are resistant to its detrimental effects and have developed the ability to degrade it during previous exposure to the compound.

Generally, the biodegradation of all three antibiotics in the estuarine water is weak, as demonstrated by the removal efficiency of the three antibiotics via biodegradation ranging from 6.2 to $8.0 \%$ (Fig. 6). A comparison of the removal efficiency of antibiotics through biodegradation in different mediums is shown in Table S5. Our results were similar to the removal efficiency of sulfonamides in domestic sewage but significantly lower than those in activated sludge and sediment. Kummerer (2010) reported that the microbial density in wastewater and river water is lower than that in the corresponding activated sludge and sediment. This may be one of the reasons for the lower biodegradation of antibiotics in aqueous media. Another possible reason may be that the tested antibiotics are the only source of carbon for microbiota. Several studies have reported that the biodegradation of

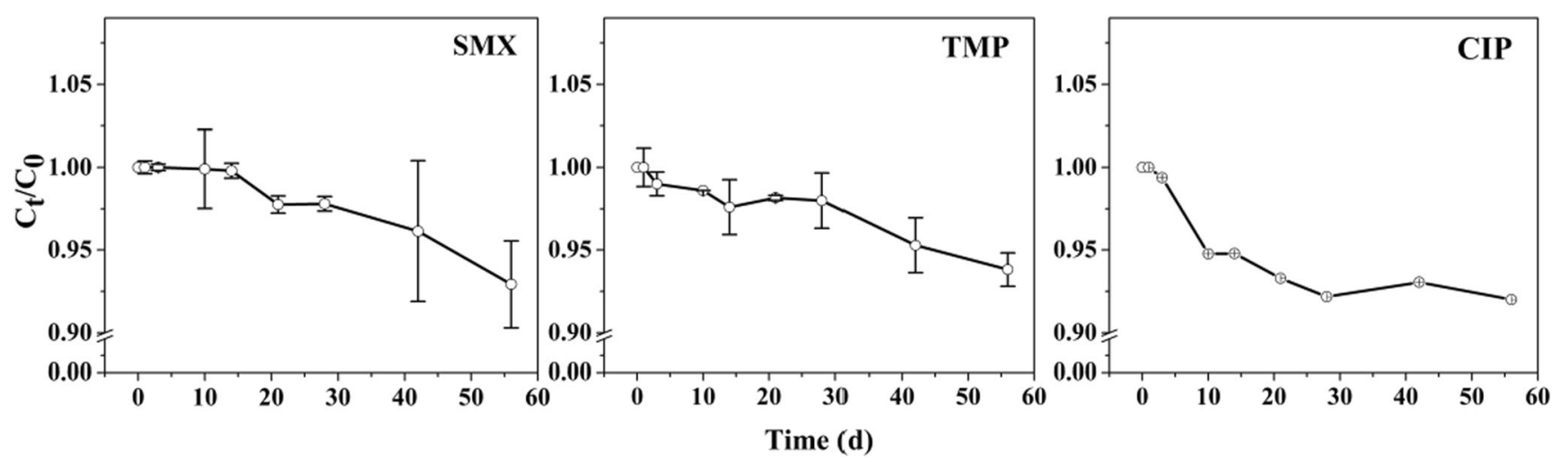

Fig. 4 Biodegradation of antibiotics in the estuarine water 
antibiotics can be enhanced by a co-metabolism mechanism (Alexy et al. 2004; Xu et al. 2011). Thus, the removal efficiency of antibiotics via biodegradation in activated sludge with a high carbon source concentration was higher. Although both in aqueous media, the removal efficiencies obtained in our study were lower than those reported by Patrolecco et al. (2018). This could be related to the low initial concentrations $(500 \mu \mathrm{g} / \mathrm{L})$ of the tested antibiotics in their study. Another possible reason may be the high conductivity of the estuarine water used in our study. Adamek et al. (2016) highlighted that high concentration of salts in river water can inhibit the biodegradation of sulfonamides.

\section{Multiple reactions of antibiotics}

In the natural water environment, the hydrolysis, photolysis, and biodegradation of antibiotics occur simultaneously. Simulating the aqueous concentrations of antibiotics when multiple reactions occur simultaneously can more realistically reveal their decay rate constants. Multiple reactions of the three antibiotics in the estuarine water were studied, and the results are shown in Fig. 5. After 56 days of degradation, approximately $80 \%$ of the CIP was degraded. For SMX and TMP, the decay efficiencies were $36.0 \%$ and $22.2 \%$, respectively. Clearly, the removal efficiencies of the three antibiotics under multiple reactions were greater than those in any single reaction. Among the different antibiotics, CIP was more prone to degradation, followed by SMX, while TMP was relatively stable in the natural water environment, indicating that the decay efficiencies of the antibiotics were related to their structures and properties. Our results were in accordance with a previous study which reported that TMP is refractory in seawater (Alexy et al. 2004). The multiple decay processes of the three antibiotics conformed to the first-order kinetic reaction model $\left(R^{2}>\right.$ 0.95). As shown in Table 2, the multiple degradation rate constants of SMX, TMP, and CIP are $9.15 \times 10^{-3} \pm 5.29 \times 10^{-4} / \mathrm{d}$, $4.89 \times 10^{-3} \pm 1.70 \times 10^{-4} / \mathrm{d}$, and $2.84 \times 10^{-2} \pm 1.18 \times 10^{-3} / \mathrm{d}$, respectively. The combined degradation rates were higher than the algebraic sum of the decay rates of the single reactions (i.e., hydrolysis, photolysis, and biodegradation). This indicated that there may be synergistic effects between the various reaction processes. The synergistic effects of photodegradation and biodegradation in determining the disappearance of SMX have been demonstrated by Patrolecco et al. (2018). Therefore, it is more scientific to obtain degradation rate parameters of antibiotics by conducting multiple reaction experiments.

\section{Evaluation of various environmental behaviors}

Both sorption and degradation reactions can result in the reduction in aqueous concentrations of antibiotics in natural water. However, the contribution of each reaction process to the total attenuation varies among antibiotics. In order to reveal the key attenuation processes of the target antibiotics in the estuarine water, the results obtained from single reactions and multiple reactions were compared. According to the results of the hydrolysis experiments, hydrolysis of CIP and TMP was negligible at a $\mathrm{pH}$ of 4.0, 7.0, and 9.0. Although weak hydrolysis of SMX occurred at a $\mathrm{pH}$ of 4.0, its hydrolysis process could be ignored in the natural estuarine environment with $\mathrm{pH}$ values of 7.4-8.1. Thus, it is safe to conclude that hydrolysis has no contribution to the attenuation of CIP, TMP, and SMX in the estuarine water.

All three antibiotics are biodegradable in the estuarine water. However, the removal efficiency of CIP, TMP, and SMX through biodegradation was relatively low, ranging from 6.2 to $8.0 \%$. Meanwhile, the biodegradation rates of the tested antibiotics in the estuarine water are also slow, with the corresponding half-life values ranging from 344.8 to 653.9 days (Table 2). This indicated that biodegradation contributed less to the overall attenuation of the target antibiotics. The removal efficiencies of SMX, TMP, and CIP through photolysis were 4.3, 2.0, and 9.3 times greater than those via biodegradations, respectively. The photolysis half-lives of the target antibiotics are also much shorter than the half-lives of biodegradation (Table 2). This demonstrated that photolysis plays an important role in the attenuation of antibiotics whenever the rate of biodegradation or hydrolysis is low or negligible.
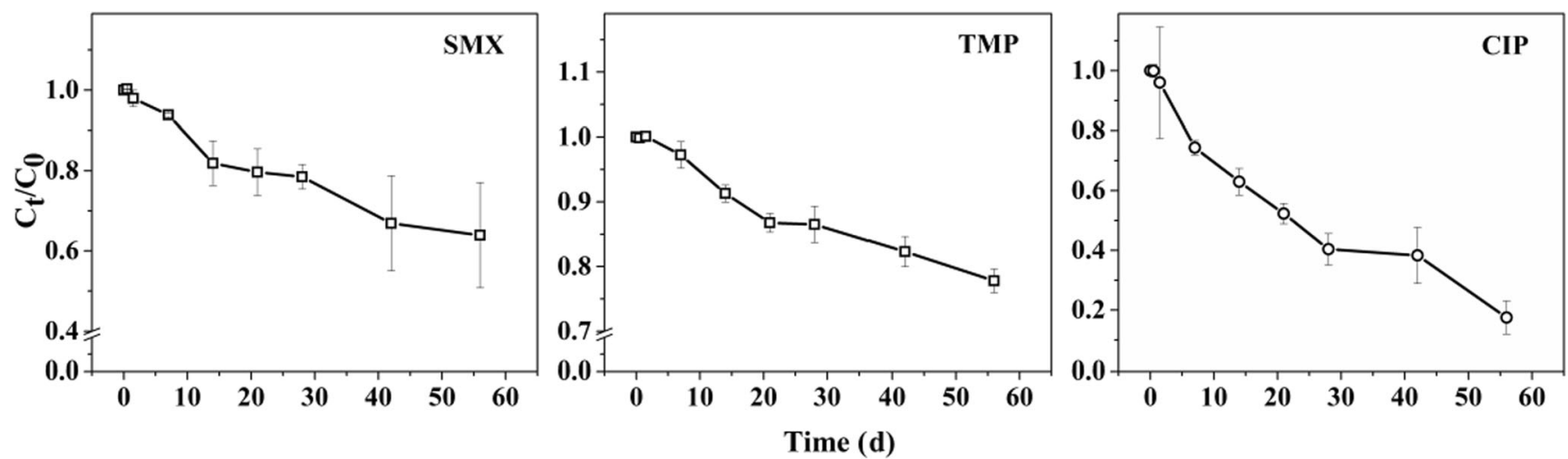

Fig. 5 Multiple reactions of antibiotics in the estuarine water 


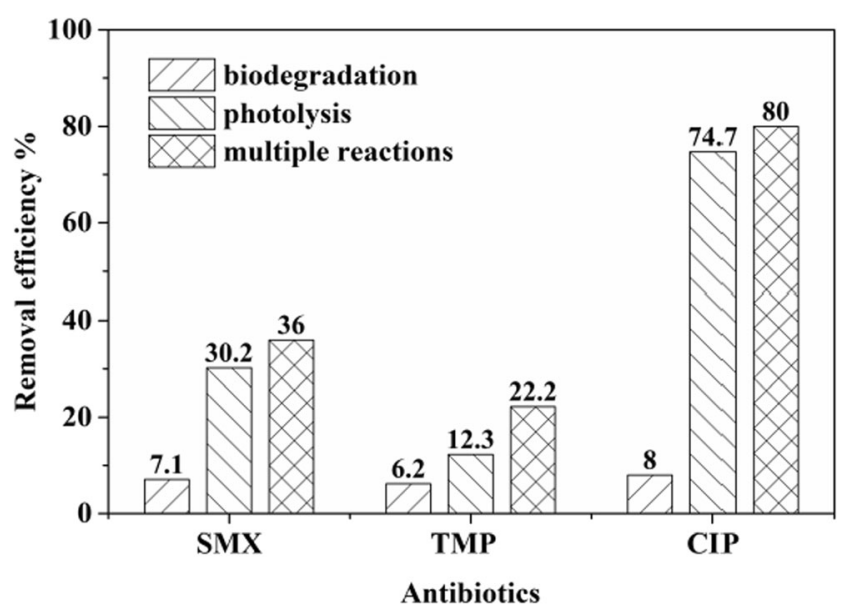

Fig. 6 Removal efficiency of antibiotics through the single reaction and multiple reactions, respectively

As shown in Fig. 6, the removal efficiency of the three antibiotics through multiple reactions is the largest and nearly equal to the sum of the removal efficiency through photolysis and biodegradation. Meanwhile, the photolysis of SMX, TMP, and CIP accounted for $83.9 \%, 55.4 \%$, and $93.4 \%$ of their overall degradation, respectively. This showed that photolysis was the key degradation process of the three antibiotics in the estuarine water. Our results were in accordance with other studies that reported that photolysis is a significant process for decreasing the concentrations of antibiotics in natural waters (Lin et al. 2010; Carstens et al. 2013; Caracciolo et al. 2018). Therefore, parameters related to the photolysis reaction should be focused on during modeling. Another key thing to note is that antibiotics may accumulate in the middle and deep waters where the solar radiation is weak.

The sorption experiments showed that all three antibiotics could be absorbed by the sediment, and the sorption rates were relatively fast. In particular, CIP reached sorption equilibrium within $6 \mathrm{~h}$. Although the time of reaching equilibrium for SMX and TMP was longer, it only required 2 days. This showed that the sorption rates of the antibiotics were much faster than their degradation rates. Therefore, when the three antibiotics entered the estuarine water, their concentrations decreased rapidly owing to sorption. After, a relatively slow photolysis and biodegradation reaction occurred, which further attenuated the concentrations of the antibiotics in environmental waters. The same results were also reported by Carstens et al. (2013), who found that photodegradation and sorption to sediment control the aqueous concentrations of sulfamethazine.

Based on the experimental data, the attenuation rates of antibiotics were related to their properties. Among the three target antibiotics, CIP was more likely to be adsorbed by sediment particles and more prone to photodegradation and biodegradation. This could be attributed to its special structure (e.g., piperazine ring) and physicochemical properties (e.g., $\mathrm{pKa})$. However, it has to be considered that CIP removal in surface water is offset because this antibiotic is continuously discharged through various sources and can also be desorbed, thereby leading to pseudo-persistence. In contrast, the extremely symmetric structure of TMP makes it relatively stable in water. This may explain why the maximum concentration of TMP in the XQR estuary is up to the $\mu \mathrm{g} / \mathrm{L}$ level ( $\mathrm{Li}$ et al. 2020). Furthermore, environmental factors also have effects on the attenuation of antibiotics in estuarine environments. This topic should be addressed in future studies.

\section{Conclusions}

In this study, the single reactions (i.e., sorption, hydrolysis, photolysis, and biodegradation) and multiple reactions of SMX, TMP, and CIP in aquatic systems were studied. Hydrolysis and biodegradation had no or a low contribution to the degradation of the target antibiotics in the estuarine water. Photolysis was the key degradation process for SMX, TMP, and CIP in water. Compared with that in ultrapure water, the photolysis of the tested antibiotics was more prone to occur in the estuarine water. The multiple reaction rates were higher than the algebraic sum of the single reaction rates, implying that there may be synergistic effects between the various reaction processes. Furthermore, the rate of degradation of the three antibiotics in the estuarine water was in the order of CIP > SMX > TMP. The sorption experiments indicated that the sorption rates of the target antibiotics onto sediments with different properties were very fast. By comparing the rates of various single reactions, we concluded that the antibiotic concentrations decreased rapidly by sorption after entering the estuarine water and then decreased relatively slowly through photolysis and biodegradation reactions. Additionally, antibiotics may accumulate in the middle and deep estuarine water, the aphotic zone. Future studies are needed to determine whether this process will alter the vertical distribution characteristics of antibiotic concentrations.

Funding information This study was financially supported by Shandong Key Laboratory of Coastal Environmental Processes, YICCAS (2019SDHADKFJJ12), and the Natural Science Foundation of the Jiangsu Higher Education Institutions of China (19KJB610007).

\section{References}

Adamek E, Baran W, Sobczak A (2016) Assessment of the biodegradability of selected sulfa drugs in two polluted rivers in Poland: effects of seasonal variations, accidental contamination, turbidity and salinity. J Hazard Mater 313:147-158 
Alexy R, Kumpel T, Kummerer K (2004) Assessment of degradation of 18 antibiotics in the closed bottle test. Chemosphere 57:505-512

Alharbi SK, Kang J, Nghiem LD, van de Merwe JP, Leusch FDL, Price WE (2017) Photolysis and $\mathrm{UV} / \mathrm{H}_{2} \mathrm{O}_{2}$ of diclofenac, sulfamethoxazole, carbamazepine, and trimethoprim: identification of their major degradation products by ESI-LC-MS and assessment of the toxicity of reaction mixtures. Process Saf Environ 112:222-234

Amorim CL, Moreira IS, Maia AS, Tiritan ME, Castro PML (2014) Biodegradation of ofloxacin, norfloxacin, and ciprofloxacin as single and mixed substrates by Labrys portucalensis F11. Appl Microbiol Biotechnol 98:3181-3190

Arlos MJ, Bragg LM, Servos MR, Parker WJ (2014) Simulation of the fate of selected pharmaceuticals and personal care products in a highly impacted reach of a Canadian watershed. Sci Total Environ 485:193-204

Bialk-Bielinska A, Stolte S, Arning J, Uebers U, Boschen A, Stepnowski P, Matzke M (2011) Ecotoxicity evaluation of selected sulfonamides. Chemosphere 85:928-933

Bialk-Bielinska A, Stolte S, Matzke M, Fabianska A, Maszkowska J, Kolodziejska M, Liberek B, Stepnowski P, Kumirska J (2012) Hydrolysis of sulphonamides in aqueous solutions. J Hazard Mater 221:264-274

Biel-Maeso M, Baena-Nogueras RM, Corada-Fernandez C, Lara-Martin PA (2018) Occurrence, distribution and environmental risk of pharmaceutically active compounds (PhACs) in coastal and ocean waters from the Gulf of Cadiz (SW Spain). Sci Total Environ 612:649659

Biosic M, Mitrevski M, Babic S (2017) Environmental behavior of sulfadiazine, sulfamethazine, and their metabolites. Environ Sci Pollut Res 24:9802-9812

Cao X, Pang H, Yang G (2015) Sorption behaviour of norfloxacin on marine sediments. J Soils Sediments 15:1635-1643

Caracciolo AB, Grenni P, Rauseo J, Ademollo N, Cardoni M, Rolando L, Patrolecco L (2018) Degradation of a fluoroquinolone antibiotic in an urbanized stretch of the River Tiber. Microchem J 136:43-48

Cardoza LA, Knapp CW, Larive CK, Belden JB, Lydy M, Graham DW (2005) Factors affecting the fate of ciprofloxacin in aquatic field systems. Water Air Soil Pollut 164:383-398

Carstens KL, Gross AD, Moorman TB, Coats JR (2013) Sorption and photodegradation processes govern distribution and fate of sulfamethazine in freshwater-sediment microcosms. Environ Sci Technol 47:10877-10883

Chapman PM, Wang FY (2001) Assessing sediment contamination in estuaries. Environ Toxicol Chem 20:3-22

Charuaud L, Jarde E, Jaffrezic A, Thomas MF, Le Bot B (2019) Veterinary pharmaceutical residues from natural water to tap water: sales, occurrence and fate. J Hazard Mater 361:169-186

Cheng J, Jiang L, Sun T, Tang Y, Du Z, Lee L, Zhao Q (2019) Occurrence, seasonal variation and risk assessment of antibiotics in the surface water of North China. Arch Environ ConTox 77:8897

Choi YJ, Kim LH, Zoh KD (2016) Removal characteristics and mechanism of antibiotics using constructed wetlands. Ecol Eng 91:85-92

Fernandez-Calvino D, Bermudez-Couso A, Arias-Estevez M, NovoaMunoz JC, Fernandez-Sanjurjo MJ, Alvarez-Rodriguez E, NunezDelgado A (2015) Kinetics of tetracycline, oxytetracycline, and chlortetracycline adsorption and desorption on two acid soils. Environ Sci Pollut Res 22:425-433

Garcia-Galan MJ, Diaz-Cruz MS, Barcelo D (2012) Kinetic studies and characterization of photolytic products of sulfamethazine, sulfapyridine and their acetylated metabolites in water under simulated solar irradiation. Water Res 46:711-722

Gmurek M, Horn H, Majewsky M (2015) Phototransformation of sulfamethoxazole under simulated sunlight: transformation products and their antibacterial activity toward Vibrio fischeri. Sci Total Environ 538:58-63
Gobel A, McArdell CS, Suter MJ, Giger W (2004) Trace determination of macrolide and sulfonamide antimicrobials, a human sulfonamide metabolite, and trimethoprim in wastewater using liquid chromatography coupled to electrospray tandem mass spectrometry. Anal Chem 76:4756-4764

Guerra P, Kim M, Shah A, Alaee M, Smyth SA (2014) Occurrence and fate of antibiotic, analgesic/anti-inflammatory, and antifungal compounds in five wastewater treatment processes. Sci Total Environ 473:235-243

Homem V, Santos L (2011) Degradation and removal methods of antibiotics from aqueous matrices - a review. J Environ Manag 92:2304 2347

Hosseini NA, Parker WJ, Matott LS (2012) Modelling concentrations of pharmaceuticals and personal care products in a Canadian watershed. Can Water Resour J 37:191-208

Huijbers PMC, Larsson DGJ, Flach CF (2020) Surveillance of antibiotic resistant Escherichia coli in human populations through urban wastewater in ten European countries. Environ Pollut 261:114200

Ingerslev F, Halling-Sorensen B (2000) Biodegradability properties of sulfonamides in activated sludge. Environ Toxicol Chem 19: 2467-2473

Jia Y, Khanal SK, Shu H, Zhang H, Chen GH, Lu H (2018) Ciprofloxacin degradation in anaerobic sulfate-reducing bacteria (SRB) sludge system: mechanism and pathways. Water Res 136:64-74

Kummerer K (2010) Pharmaceuticals in the environment. Annu Rev Environ Resour 35:57-75

Li J, Cui M, Zhang H (2020) Spatial and temporal variations of antibiotics in a tidal river. Environ Monit Assess 192(336):336

Li J, Zhang H (2016) Adsorption-desorption of oxytetracycline on marine sediments: kinetics and influencing factors. Chemosphere 164:156 163

Li J, Zhang H (2017) Factors influencing adsorption and desorption of trimethoprim on marine sediments: mechanisms and kinetics. Environ Sci Pollut Res 24:21929-21937

Li J, Zhang KN, Zhang H (2018) Adsorption of antibiotics on microplastics. Environ Pollut 237:460-467

Li WH, Shi YL, Gao LH, Liu JM, Cai YQ (2012) Occurrence of antibiotics in water, sediments, aquatic plants, and animals from Baiyangdian Lake in North China. Chemosphere 89:1307-1315

Lin AYC, Reinhard M (2005) Photodegradation of common environmental pharmaceuticals and estrogens in river water. Environ Toxicol Chem 24:1303-1309

Lin JS, Pan HY, Liu SM, Lai HT (2010) Effects of light and microbial activity on the degradation of two fluoroquinolone antibiotics in pond water and sediment. J Environ Sci Health B 45:456-465

Liu SS, Zhao HX, Lehmler HJ, Cai X, Chen J (2017) Antibiotic pollution in marine food webs in Laizhou Bay, North China: trophodynamics and human exposure implication. Environ Sci Technol 51:23922400

Loftin KA, Adams CD, Meyer MT, Surampalli R (2008) Effects of ionic strength, temperature, and $\mathrm{pH}$ on degradation of selected antibiotics. J Environ Qual 37:378-386

Luo Y, Mao DQ, Rysz M, Zhou QX, Zhang HJ, Xu L, Alvarez PJJ (2010) Trends in antibiotic resistance genes occurrence in the Haihe River, China. Environ Sci Technol 44:7220-7225

Ngigi AN, Magu MM, Muendo BM (2020) Occurrence of antibiotics residues in hospital wastewater, wastewater treatment plant, and in surface water in Nairobi County, Kenya. Environ Monit Assess 192(1)

Norvill ZN, Shilton A, Guieysse B (2016) Emerging contaminant degradation and removal in algal wastewater treatment ponds: identifying the research gaps. J Hazard Mater 313:291-309

Patrolecco L, Rauseo J, Ademollo N, Grenni P, Cardoni M, Levantesi C, Luprano ML, Caracciolo AB (2018) Persistence of the antibiotic sulfamethoxazole in river water alone or in the co-presence of ciprofloxacin. Sci Total Environ 640:1438-1446 
Riaz L, Mahmood T, Khalid A, Rashid A, Siddique MBA, Kamal A, Coyne MS (2018) Fluoroquinolones (FQs) in the environment: a review on their abundance, sorption and toxicity in soil. Chemosphere 191:704-720

Schowanek D, Webb S (2002) Exposure simulation for pharmaceuticals in European surface waters with GREAT-ER. Toxicol Lett 131:39 50

Simpson JH, Vennell R, Souza AJ (2001) The salt fluxes in a tidallyenergetic estuary. Estuar Coast Shelf S 52:131-142

Tong L, Eichhorn P, Perez S, Wang Y, Barcelo D (2011) Photodegradation of azithromycin in various aqueous systems under simulated and natural solar radiation: kinetics and identification of photoproducts. Chemosphere 83:340-348

Van Boeckel TP, Brower C, Gilbert M, Grenfell BT, Levin SA, Robinson TP, Teillant A, Laxminarayan R (2015) Global trends in antimicrobial use in food animals. P Natl Acad Sci USA 112:5649-5654

Wang L, Yang L, Li Y, Zhang Y, Ma X, Ye Z (2010) Study on adsorption mechanism of $\mathrm{Pb}(\mathrm{II})$ and $\mathrm{Cu}$ (II) in aqueous solution using PS-EDTA resin. Chem Eng J 163:364-372

Xu B, Mao D, Luo Y, Xu L (2011) Sulfamethoxazole biodegradation and biotransformation in the water-sediment system of a natural river. Bioresour Technol 102:7069-7076
Zhang C, Hu XF, Luo YM (2016) Aqueous photodegradation of oxytetracycline under simulated sunlight irradiation. Huanjing HuaxueEnviron Chem 35:430-438

Zhang RJ, Tang JH, Li J, Zheng Q, Liu D, Chen YJ, Zou YD, Chen XX, Luo CL, Zhang G (2013) Antibiotics in the offshore waters of the Bohai Sea and the Yellow Sea in China: occurrence, distribution and ecological risks. Environ Pollut 174:71-77

Zhu YG, Johnson TA, Su JQ, Qiao M, Guo GX, Stedtfeld RD, Hashsham SA, Tiedje JM (2013) Diverse and abundant antibiotic resistance genes in Chinese swine farms. P Natl Acad Sci USA 110:34353440

Zhuang J, Wang S, Tan Y, Xiao R, Chen J, Wang X, Jiang L, Wang Z (2019) Degradation of sulfadimethoxine by permanganate in aquatic environment: influence factors, intermediate products and theoretical study. Sci Total Environ 671:705-713

Publisher's note Springer Nature remains neutral with regard to jurisdictional claims in published maps and institutional affiliations. 